$34.8 \%, \mathrm{p}=0.02)$ had lower rates of DM $(12 \%$ vs $24.9 \%$, $\mathrm{p}=0.047)$ and pre-procedure glucose levels $(114[104-143] \mathrm{mg} /$ $\mathrm{dl}$ vs 126[109-152], $\mathrm{p}=0.013)$. Other baseline characteristics and procedural parameters were comparable between groups. In terms of outcomes, rates of successful reperfusion (mTICI 2b-3) $(92 \% \%$ vs $91.9 \%, \mathrm{p}=1.0)$ and any parenchymal hematomas $(\mathrm{PH})(8.3 \%$ vs $13.3 \%, \mathrm{p}=0.34)$ were comparable. There was a non-significant trend toward better 90-Day independent outcomes (mRS 0-2) and higher 90-day mortality favoring octogenarians $(13.6 \%$ vs $25.3 \%, p=0.094$ and $52.3 \%$ vs $38.7 \%, p=0.095$ respectively) while ambulatory outcomes were more similar (mRS $0-3)(38.1 \%$ vs $41.9 \%, p=0.212)$. There was a non-significant shift in the overall distribution of 90-day mRS favoring octogenarians. In multivariate analysis, baseline NIHSS, ASPECTS, IV t-PA, Successful reperfusion (mTICI 2b-3) and any $\mathrm{PH}$ were independent predictors of a favorable shift in mRS while age category was not. A FIV $\leq 16.9 \mathrm{ml}$ (sensitivity $67 \%$, specificity $75 \%$ ) in octogenarians and less than $12.8 \mathrm{~mL}$ (sensitivity $79 \%$, specificity $80 \%$ ) in nonagenarians demonstrated the greatest accuracy for identifying good outcomes.

Conclusions Our study shows that there were no differences in procedural and clinical outcomes between nonagenarians and octogenarians treated with ET with more than $1 / 3$ of nonagenarians were ambulatory at 90 days. However, advanced age seems to be associated with reduced tolerance for infarct volume. Further research is needed to optimize selection modalities in this age cohort.

Disclosures M. Bouslama: None. G. Rodrigues: None. L. Pisani: None. D. Haussen: None. M. Frankel: None. R. Nogueira: 2; C; Covidien/Medtronic (SWIFT and SWIFTPRIME Steering Committee - modest, STAR Trial Core Lab significant), Stryker Neurovascular (Trevo-2 Trial PI - modest, DAWN Trial PI - no payment, Trevo Registry Steering Committee - no payment, Physician Advisory Board/Consultant significant), Neuravi/Cerenovus (ARISE-II Trial Steering Committee - no payment, Physician Advisory Board - modest), Phenox (Physician Advisory Board/Consultant - modest), Genentech (Physician Advisory Board/Consultant - modest). 6; C; Penumbra (3-D Separator Trial Executive Committee - no payment).

\section{E-142 EFFICACY AND SAFETY OF MECHANICAL THROMBECTOMY USING LARGER BORE JET 7 ASPIRATION CATHETERS FOR INTRACRANIAL LARGE VESSEL OCCLUSION}

${ }^{1}$ A Hassan* ${ }^{*}{ }^{2} \mathrm{~J}$ Fifi, ${ }^{3} \mathrm{O}$ Zaidat. 'Valley Baptist Medical Center, Harlingen, $T X ;{ }^{2}$ The Mount Sinai Hospital, New York, NY; ${ }^{3}$ Mercy Health St. Vincent Medical Center, Toledo, $\mathrm{OH}$

10.1136/neurintsurg-2019-SNIS.217

Purpose To report initial experience of A Direct Aspiration first-Pass Thrombectomy (ADAPT) technique using larger bore JET 7 aspiration catheters in patients experiencing acute ischemic stroke secondary to intracranial large vessel occlusions (LVO).

Materials and methods COMPLETE is a prospective, singlearm, multi-center observational registry, including patients with intracranial LVO who are eligible for mechanical thrombectomy and have planned frontline treatment with Penumbra System. We performed an interim analysis in patients treated with new larger bore JET 7 aspiration catheters (inner diameter of 0.072 '). Primary outcomes included angiographic revascularization of the occluded target vessel at immediate post-procedure as defined by an mTICI $2 b$ or higher, functional outcome at 90 days post-procedure as defined by a mRS 0-2, and all-cause mortality at 90 days. Secondary outcomes included incidence of device- and procedure-related serious adverse events (SAEs), occurrence of embolization in previously uninvolved (or new) territories (ENT), and occurrence of symptomatic intracranial hemorrhages (sICH) at 24 hours.

Results Fifty-five patients (mean age $66.5 \pm 13.0 ; 50.9 \%$ female) treated at 12 sites in the US were included in this analysis. Baseline NIHSS (mean \pm SD) was $14.8 \pm 7.3$. Median ASPECT Score was 9.0 (IQR, 8.0-10.0). Target vessel location was the internal carotid artery or terminus in $18.2 \%$ (10/55), M1 in 52.7\% (29/55), M2 in $16.4 \%$ (9/55), and posterior circulation in $12.7 \%$ (7/55) of patients. Median procedure time was 28.0 minutes (IQR, 16.0-50.0).

Successful revascularization (mTICI $2 b$ or higher) was achieved in $92.6 \%(50 / 54)$ of patients. Good functional outcome (mRS 0-2) at 90 days was observed in $42.9 \%(6 / 14)$ of patients; follow-up data collection is ongoing. Mortality occurred in $10.9 \%(6 / 55)$ of patients. Procedure- and devicerelated SAEs occurred in 5.6\% (3/54) and $1.9 \%(1 / 54)$ of patients, respectively. Vessel dissection was reported in $1.9 \%$ (1/54) of patients. There were no occurrences of sICH, ENT, or vessel perforation.

Conclusion In this initial experience, the safety and efficacy of Penumbra System with larger bore JET 7 aspiration catheters for patients with LVO is acceptable and comparable to other aspiration catheters. Use of larger bore aspiration catheters resulted in fast overall procedural time.

Disclosures A. Hassan: 1; C; Penumbra. 2; C; Penumbra, GE Healthcare, Medtronic, Stryker, MicroVention, Genentech. 3; C; Penumbra, GE Healthcare, Medtronic, Stryker, MicroVention, Genentech. J. Fifi: 1; C; Penumbra. 2; C; Penumbra. O. Zaidat: 1; C; Penumbra, Stryker, Genentech, Medtronic Neurovascular. 2; C; Penumbra, Medtronic Neurovascular, Codman, Stryker.

\section{E-143 SHORT-TERM IN-HOSPITAL OUTCOMES OF THROMBOLYSIS FOR ACUTE ISCHEMIC STROKE PATIENTS WITH NON-PRIMARY BRAIN TUMORS AND PERIPHERAL VASCULAR DISEASE}

K Tong*, A Wang-Selfridge, P Brauer. Anatomy, Kansas City University of Medicine and Biosciences, Kansas City, MO

10.1136/neurintsurg-2019-SNIS.218

Background Intravenous thrombolysis remains an underutilized treatment for acute ischemic stroke (AIS) due to several relative and absolute contraindications. Previous studies have found similar outcomes after thrombolysis between AIS patients with benign brain tumors and AIS patients without. This study aims to investigate short-term outcomes of thrombolytic treatment for the greater majority of AIS patients who have no history of primary brain tumors, particularly those with peripheral vascular disease (PVD).

Methods This retrospective cohort study utilized data from the 2012-2015Q3 Nationwide Inpatient Sample (NIS). ICD-9 codes identified adult patients (ages 18+) who suffered acute ischemic stroke and received intravenous thrombolysis, and then further 